\title{
Das E-Assessment-Tool DMT
}

\section{Ein Werkzeug zur automatischen Bewertung und Feedback-Generierung für typische Übungsaufgaben im Fachbereich Datenbanken}

\author{
Andreas Thor ${ }^{1}$ (D) - Toralf Kirsten ${ }^{2}$ \\ Eingegangen: 11. Oktober 2020 / Angenommen: 5. Januar 2021 / Online publiziert: 24. Januar 2021 \\ (c) Der/die Autor(en) 2021
}

\section{Zusammenfassung}

Die Bearbeitung von Übungsaufgaben ist ein wichtiges Element in der Datenbank-Lehre. Lösungen der Studierenden lassen sich dabei häufig in strukturierten Ergebnisformaten festhalten, wie z.B. SQL-Anfragen oder die Spezifikation von Schemata und Relationen. Dieser Beitrag stellt das E-Assessment-Tool DMT (Data Management Tester) vor, das sowohl eine automatische Bewertung als auch eine automatische Feedback-Generierung solcher strukturierter Lösungen ermöglicht. Es soll dabei insbesondere Studierende unterstützen, nicht ganz korrekte Lösungen zielgerichtet überarbeiten zu können. Dieser Betrag skizziert Konzept und Architektur von DMT und erläutert den Einsatz an der HTWK Leipzig und der Hochschule Mittweida.

Schlüsselwörter E-Assessment $\cdot \mathrm{SQL} \cdot$ Datenbanklehre

\section{Einführung}

Die regelmäßige Bearbeitung von Übungsaufgaben durch Studierende ist ein zentrales Element für den Lernerfolg (nicht nur) in MINT-Fächern. Nach der Abgabe der Lösungen sollte eine zeitnahe Bewertung inklusive Feedback erfolgen, damit Studierende eine regelmäßige Einschätzung ihres Leistungsstandes erhalten und auf Grund eventuell gemachter Fehler ihre Lernarbeit anpassen können. Die manuelle Bewertung von Lösungen sowie die Erstellung eines Feedbacks, das u. a. angibt, welche Fehler bzw. Arten von Fehlern gemacht worden sind, resultieren in einem hohen Arbeitsaufwand für die Lehrenden insbesondere bei großen Studiengruppen.

Andreas Thor

andreas.thor@htwk-leipzig.de

Toralf Kirsten

toralf.kirsten@hs-mittweida.de

1 Hochschule für Technik, Wirtschaft und Kultur Leipzig, Leipzig, Deutschland

2 Hochschule Mittweida, Mittweida, Deutschland
Zur Reduktion dieser Zeitaufwände hat sich in den letzten Jahren E-Assessment etabliert, welches standardisierte Aufgabenformate anbietet, die größtenteils automatisch auswertbar sind. Gängige Lern-Management-Systeme wie Ilias $^{1}$, Moodle $^{2}$ oder OLAT ${ }^{3}$ bieten dazu Lehrenden u. a. die Möglichkeit, Single- und Multiple-Choice-Aufgaben sowie Aufgaben zur Bestimmung von Zuordnungen oder Reihenfolgen zu definieren. Dabei wird neben Aufgabenstellung und Antwortoptionen zusätzlich die korrekte Lösung spezifiziert, welche dann für die automatische Bewertung der studentischen Lösungen genutzt wird. Zusätzlich kann ein zuvor hinterlegtes, standardisiertes Feedback angezeigt werden, welches z. B. bei der Auswahl einer falschen Antwortoption einer Multiple-Choice-Frage erläutert, warum die gewählte Option falsch ist.

E-Assessment mit den erwähnten standardisierten Aufgabenformaten lässt sich natürlich auch in der DatenbankLehre einsetzen. Neben dem Abfragen von Faktenwissen (Kompetenzstufe Erinnern in der Taxonomie nach Anderson und Krathwohl [5]) erlauben z. B. Multiple-Choice-Fragen auch die Prüfung von Kompetenzen der Taxonomiestu-

\footnotetext{
1 https://www.ilias.de/.

2 https://moodle.de.

3 https://olat.org/.
} 
fen Verstehen und Anwenden. Ein Beispiel wäre die Frage „Welche der folgenden Tupel sind im Ergebnis der Query SELECT a,b FROM R WHERE $c>5$ enthalten?", bei der zusätzlich eine Relation $R$ angegeben wird und die Antwortoptionen aus Tupeln bestehen. Damit lässt sich z. B. das Verständnis von SQL-Queries überprüfen.

Die Nutzung standardisierter Aufgabenformate für die Datenbank-Lehre hat jedoch drei gravierende Nachteile. Erstens schränkt die Spezifikation von Antwortoptionen den Lösungsraum stark ein und ermöglicht ein Raten bzw. Ausschlussverfahren seitens der Studierenden. Im Beispiel der Multiple-Choice-Frage können Studierende aus den Tupeln zufällig wählen und offensichtlich unsinnige Antwortoptionen, die z. B. Attributwerte enthalten, die in R nicht vorkommen, aussschließen. Zweitens ist die Bewertung gegebener Artefakte wie z.B. einer SQL-Query oder eines Relationenschemas im Allgemeinen einfacher als deren selbständige Erstellung. Im oben genannten Beispiel muss der Studierende z.B. die korrekte Reihenfolge der Schlüsselworte SELECT, FROM und WHERE nicht wissen, da ja bereits eine syntaktisch korrekte Query Teil der Aufgabenstellung ist. Bei der selbständigen Erstellung einer Query, z. B. „Alle Tupel $(\mathrm{a}, \mathrm{b})$ aus $\mathrm{R}$, bei denen c größer als 5 ist!“”, ist dieses Wissen aber notwendig. Drittens sind die Möglichkeiten für ein Feedback bei falschen Lösungen begrenzt. Bei der Beispiel-Aufgabe kann für falsche Tupel erklärt werden, warum sie nicht Teil des Anfrageergebnisses sind. Ein Feedback, was zur selbständigen Verbesserung bzw. Korrektur der studentischen Lösung führt, kann aber nicht gegeben werden, da in diesem Moment die korrekte Lösung offenbart wird.

In den folgenden Abschnitten stellen wir das E-Assessment-Tool DMT (Data Management Tester) vor. Es erlaubt die automatische Bewertung strukturierter Ergebnisformate, die häufig als Lösung von Aufgaben in der DatenbankLehre stehen. Die Ergebnisformate umfassen u. a. SQL-Anfragen, die Spezifikation von Schemata und Relationen sowie View- und Constraint-Definitionen. Wir zeigen anhand eines exemplarischen Bachelor-Moduls „Datenbanksysteme", in welchen Themengebieten Aufgaben mit den Ergebnisformaten möglich sind und wie diese durch die Lehrenden definiert werden. Anschließend erläutern wir den Bewertungs- und Feedback-Mechanismus und skizzieren die Architektur der Implementation, ehe wir einen kurzen Abriss zu zwei Einsatzszenarien von DMT in den letzten Semestern an der HTWK Leipzig und der Hochschule Mittweida geben.

\section{Aufgaben und Ergebnisformate}

Das E-Assessment-Tool DMT erlaubt die Spezifikation von Aufgaben durch Lehrende und ist in der Lage, Lösungen von Studierenden zu bewerten und Feedback zu generieren. Jede Aufgabe hat eine eindeutige Id und wird zunächst durch einen Titel und eine Aufgabenstellung beschrieben. In Abhängigkeit vom Ergebnisformat spezifiert der Lehrende weitere Informationen, die zur automatischen Bewertung
Tab. 1 Beispiele für typische Aufgaben innerhalb eines Datenbank-Moduls inkl. Angabe des Ergebnisformats

\begin{tabular}{ll}
\hline Ergebnisformat & Beispiel einer Aufgabe (z. T. verkürzt) \\
Kapitel: Das Relationale Modell \\
TABLE & Führen Sie folgendes UPDATE-Statement aus und geben Sie die resultierende Tabelle R \\
an! & Ergänzen Sie mittels ALTER TABLE eine NOT - NULL-Bedingung für das Attribut a in \\
CHECK & R! \\
Kapitel: Datenbankanfragen mit SQL \\
SELECT & Erstellen Sie eine SQL-Query, die die Titel aller Bücher ausgibt! \\
TABLE & Wie lautet das Ergebnis der SQL-Query SELECT a FROM R WHERE b>7 für die \\
Kapitel: Umwandlung eines ER-Modells in das Relationale Modell \\
SCHEMA & Geben Sie das Schema der Relation LKW an, welches sich bei Umwandlung des gege- \\
benen ER-Modells unter Verwendung der horizontalen Partitionierung ergibt! \\
CHECK & Ergänzen Sie einen Constraint zur Relation Station, der den 1 : 1-Abbildungstyp der \\
Kapitel: Normalisierung \\
SCHEMA & Geben Sie das Schema der Relation Mitarbeiter nach Umwandlung in die dritte Normal- \\
& form an! \\
VIEW & Definieren Sie die Relation Mitarbeiter der 2NF als Sicht über die Relationen in 3NF! \\
Kapitel: Datenkontrolle &
\end{tabular}


a

Wie lautet das Ergebnis der SQL-Query SELECT $a, b, d$ FROM R JOIN $S$ ON $(b>c)$ für die Relationen $R(a, b)=\{(1,5),(2,6),(3,5),(4,7)\}$ und $S(c, d)=\{(5,3),(6,7),(8,9)\}$ ?

\begin{tabular}{|l|l|l|l|l|}
\hline $\mathbf{a}$ & $\mathbf{b}$ & $\mathbf{d}$ & & \\
\hline 2 & 6 & 3 & & \\
\hline 4 & 7 & 3 & & \\
\hline 4 & 7 & 7 & & \\
\hline & & & \\
\hline
\end{tabular}

Database ready (2 tables $[R, S]$ )

ABGABE ÜBERPRÜFEN b

Geben Sie die Titel aller Bücher aus!

Die korrekte Lösung hat 4877 Datensätze

Geben Sie Ihren SQL-Code hier ein

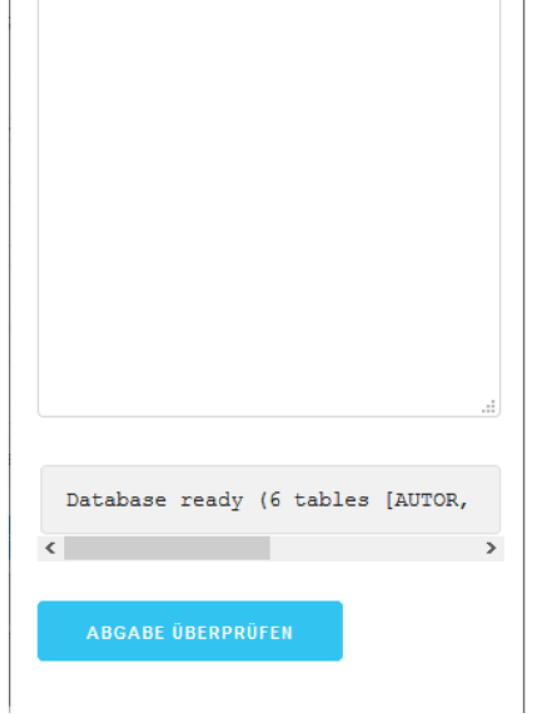

c

Geben Sie das Schema der Relation LKW an, das bei Verwendung der Horizontalen Partitionierung resultiert! Geben Sie dazu die Namen aller Attribute an und spezifizieren Sie jeweils, ob das Attribut (Teil des) Primärschlüssel(s) und/oder (Teil eines) Fremdschlüssel(s) ist!

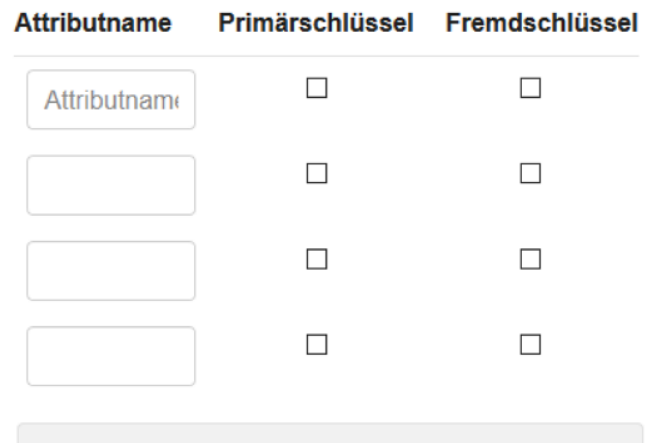

Database ready ( 3 tables [FAHRZEUG, LKW, PKW])

ABGABE ÜBERPRÜFEN

Abb. 1 Screenshot für drei verschiedene Aufgaben vom Typ TABLE (a, mit eingetragener korrekter Lösung), SELECT (b) und SCHEMA (c)

notwendig sind. Im Folgenden werden zunächst die fünf bisher unterstützten Ergebnisformate inklusive der benötigten Informationen kurz charakterisiert. Darüber hinaus nennt Tab. 1 für typische Themengebiete eines DatenbankModuls in der Hochschullehre jeweils zwei Beispielaufgaben inkl. Ergebnisformat.

- SELECT: Der Studierende gibt als Ergebnis eine SQLQuery an. Zur automatischen Bewertung spezifiziert der Lehrende eine SQL-Query als Musterlösung und gibt zusätzlich an, ob die Reihenfolge der Datensätze relevant ist. Abb. $1 b$ zeigt das User Interface einer Beispielaufgabe diesen Typs.

- SCHEMA: Der Studierende spezifiziert das vereinfachte Schema einer Relation (siehe Abb. 1c). Dazu gibt er die Attributnamen (ohne Datentypen) an und markiert für jedes Attribut, ob es ein Primärschlüssel- und/ oder Fremdschlüssel-Attribut ist (ohne Angabe der referenzierten Relation). Zum Vergleich mit der korrekten Lösung gibt der Lehrende den Namen einer Relation an, die das gesuchte Schema hat.

- TABLE: Der Studierende gibt die Attributnamen und Tupel einer Relation in Tabellenform an (siehe Abb. 1a). Zum Vergleich mit der korrekten Lösung gibt der Lehrende entweder eine SQL-Query oder das tabellarische Ergebnis im CSV-Format an. Zusätzlich kann der Lehrende spezifzieren, ob die Reihenfolge der Datensätze relevant ist, z. B. wenn das Ergebnis einer ORDER BYQuery gesucht ist.

- VIEW: Der Studierende gibt eine View-Definition als SQL-Code an. Der Lehrende spezifiziert den View-Namen sowie als Musterlösung die SQL-Query, die zur View-Definition genutzt wird.

- CHECK: Der Studierende gibt SQL-Code bestehend aus DDL-Statements, wie z.B. ALTER TABLE oder CREATE TRIGGER, an. Zur Überprüfung definiert der Lehrende ein oder mehrere SQL-Statements, für die jeweils ein definierter Datenbank-spezifischer Status- bzw. Fehlercode oder eine definierte Tupel-Menge erwartet wird.

Das in Abb. 1 gezeigte User Interface ist bewusst sehr einfach gehalten und verzichtet auf die Darstellung zusätzlicher Informationen wie z.B. einem vollständigen Schema (inkl. Attributnamen aller Relationen) bei Aufgaben des Typs SELECT (Abb. 1b) oder einem ER-Modell bei dessen Umwandlung in das relationale Modell (Abb. 1c). Diese, für die Bearbeitung der Aufgaben notwendigen Informationen, müssen von den Lehrenden durch weitere Materialien (z.B. Übungsblatt als PDF-Dokument) bereitgestellt werden. DMT dient in diesem Lehrszenario „,nur“ als Abgabebzw. Bewertungs- und Feedbacksystem der Aufgaben.

Die Spezifikation der Aufgaben erfolgt im JSON-Format. Jede Aufgabe wird durch ein JSON-Objekt mit den oben genannten Attributen spezifiziert. Aufgaben, die auf 


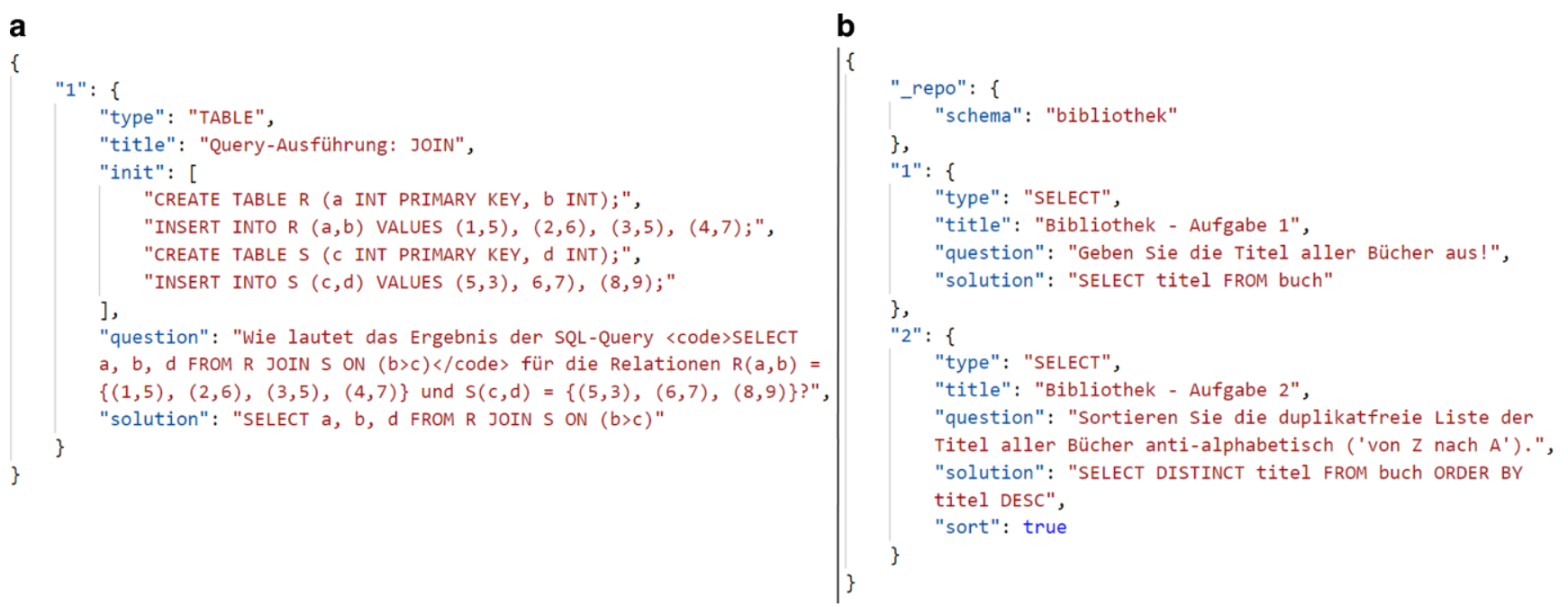

Abb. 2 Screenshot zweier Aufgaben-Repositories. Das linke Repository enthält die Definition der in Abb. 1a dargestellten Aufgabe. Das rechte Repository enthält zwei Aufgaben zum Datenbankschema Bibliothek. Die erste Aufgabe ist ebenfalls in Abb. 1b zu sehen

der selben Datenbank operieren, werden in einer als Repository bezeichneten JSON-Datei zusammengefasst. Abb. 2 zeigt zwei einfache Repositories. Jedes Repository enthält neben den Aufgaben optional den Namen des standardmäBig zu verwendenden Datenbankschemas (siehe Abb. 2b) sowie in einem extra ausgezeichneten JSON-Objekt init DDL-Statements zum Anlegen weiterer Tabellen als Datengrundlage aller Aufgaben des Repositories. Zusätzlich kann jede Aufgabe ein eigenes init-Unterobjekt definieren (siehe Abb. 2a), das dann nur für die jeweilige Aufgabe ausgeführt wird. Damit ist es möglich, sowohl mehrfach verwendete Daten in einem gemeinsamen Schema zu verwenden (wie z.B. die Mondial-Datenbank ${ }^{4}$ ) als auch Aufgaben-spezifische Daten zu nutzen, wie z.B. bei der ersten Aufgabe in Abb. 1 mit den Relationen R und S. Zur Unterstützung für die Studierenden zeigt das User Interface neben der eigentlichen Aufgabenstellung - die verfügbaren Tabellennamen zusätzlich an.

Gängige Lern-Management-Systeme ermöglichen die Aufgabenspezifikation durch eine Weboberfläche, was bei vielen Aufgaben zu einer großen „Klick-Arbeit“ für Lehrende führt. Zur Reduktion des Arbeitsaufwandes für Lehrende erfolgt die Spezifikation der Aufgaben für DMT ausschließlich per JSON-Datei (im Editor der Wahl). Dies erscheint sinnvoll, da viele Dozenten programmier-affin sind und es erfahrungsgemäß viele ähnlich gelagerte Aufgaben gibt, so dass mittels Copy \& Paste eine effiziente Erstellung unterstützt wird. Gleichzeitig ermöglicht das lesbare JSON-Format einen einfachen Austausch zwischen Lehrenden sowie eine effektive Versionskontrolle.

\footnotetext{
${ }^{4}$ https://www.dbis.informatik.uni-goettingen.de/Mondial/.
}

\section{Automatische Bewertung und Generierung von Feedback}

Wesentlicher Bestandteil von DMT ist die automatische Bewertung sowie die automatische Generierung von Feedback bzgl. der eingereichten Lösung der Studierenden. Aufgaben der Formate SELECT, TABLE und VIEW werden dabei in den folgenden drei Schritten verarbeitet.

1. Umwandlung der Studierendenlösung in Tupel-Liste: Dazu wird beim Aufgabentyp SELECT die eingegebene Query gegen den Datenbankzustand ausgeführt und das Resultset komplett eingelesen. ${ }^{5}$ Für den Aufgabentyp VIEW wird zunächst die vom Studierenden angegebene View-Definition ausgeführt und anschließend das Ergebnis der Anfrage SELECT * FROM ViewName verwendet. Lösungen des Aufgabentyps TABLE (siehe Abb. 1a) werden im CSV-Format angegeben und durch einen Parser in eine Tupel-Liste konvertiert.

2. Umwandlung der Musterlösung in Tupel-Liste: Eine als SQL-Query hinterlegte Musterlösung wird analog zur Studierendenlösung ausgeführt und konvertiert. Lösungen des Aufgabentyps TABLE können auch im CSVFormat spezifiziert werden und werden entsprechend geparst.

3. Vergleich der beiden Tupel-Listen: Beide Listen werden ohne Berücksichtigung der Reihenfolge auf Gleichheit geprüft, es sei denn, bei Aufgaben des Typs SELECT oder TABLE wurde die Relevanz der Reihenfolge markiert, da z.B. die Anfrage ein ORDER BY benötigt.

\footnotetext{
5 Zur Vermeidung von Performanzengpässen verwendet die Implementation sowohl eine maximale Laufzeit der Query als auch eine maximale Größe des Anfrageergebnisses.
} 


\begin{tabular}{|l|l|l|}
\hline $\mathbf{a}$ & $\mathbf{b}$ & \\
\hline 2 & 6 & \\
\hline 4 & 7 & \\
\hline 4 & \\
\hline 4 & 7 & \\
\hline
\end{tabular}

Database ready (2 tables [R, S]

ABGABE ÜBERPRŨFEN

Points: 0 of 1

Unterschiedliche Anzahl an

Spalten (2; erwartet: 3 ).
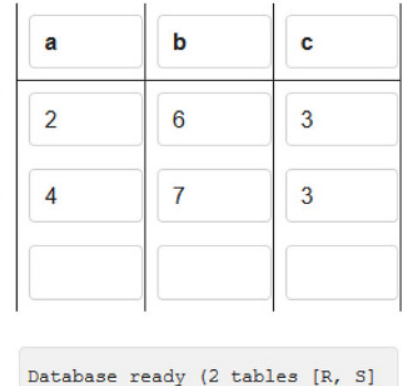

ABGABE ÜBERPRÜFEM

Points: 0 of 1

Unterschiedliche Anzahl an

Zeilen (2; erwartet: 3 ).

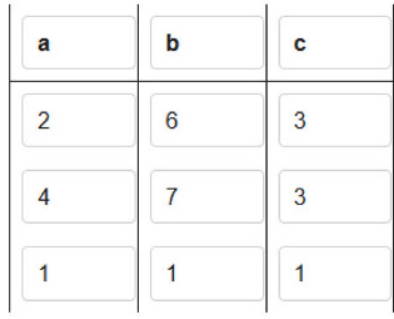

Database ready (2 tables [R, S])

ABGABE ÜBERPRÜFEM

Points: 0 of 1

Unterschiedliche Attributnamen;

falsches Attribute $C$.

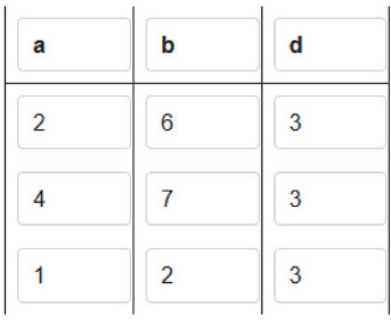

Database ready (2 tables [R, S])

ABGABE ÜBERPRÜFEN

Points: 0 of 1

Unterschiedliche Tupel:

falsches Tupel [1, 2, 3]

Abb. 3 Screenshot für vier verschiedene Arten von Feedback für die Aufgaben vom Typ TABLE aus Abb. 1a

Anhand der Prüfung kann zunächst eine Bewertung vorgenommen werden, ob die Lösung korrekt oder nicht korrekt ist. Das vorgestelle Bewertungsverfahren ist natürlich insbesondere für die Aufgabenformate SELECT und VIEW formal nicht korrekt, da die Prüfung nicht anhand der Query bzw. View-Definition erfolgt, sondern am Ergebnis bzgl. eines Datenbankzustandes. Erfahrungsgemäß spielt das aber in der Praxis kaum eine Rolle, da bei hinreichend großen Datenmengen fehlerhafte Queries meist auch zu unterschiedlichen Anfrageergebnissen führen. Ausnahmen bilden Anfragen, die eine leere Menge oder skalare Werte liefern, wie z.B. SELECT COUNT (*) FROM T. Hier würde z.B. eine Query SELECT 42 als korrekt erkannt, falls T 42 Tupel enthält.

Der Fokus von DMT liegt aber nicht auf der Bewertung sondern primär auf der Generierung individellen Feedbacks, damit Studierende animiert werden, auch bei einer falschen Lösung an der Aufgabe weiterzuarbeiten. Das Feedback resultiert aus einer strukturierten Fehleranalyse, bei der die nachfolgenden Schritte in der angegebenen Reihenfolge bearbeitet werden, wobei das Verfahren beim ersten erkannten Fehler abbricht und das generierte Feedback zurückliefert.

1. Syntax-Fehler: Die eingegebene Lösung konnte nicht ausgeführt werden und lieferte einen Syntaxfehler. Fehlermeldung und Fehlercode werden als Feedback zurückgemeldet.

2. Grad und Kardinalität: Die zu vergleichenden Relationen unterscheiden sich in Grad und/oder Kardinalität. Als Feedback werden die erwarteten Werte für die Anzahl der Spalten und die Anzahl der Zeilen zurückgegeben.

3. Schema: Die Relationen unterscheiden sich im Schema, d.h. die Menge der Attributnamen ist nicht äquivalent.
Ein falscher Attributname wird als Feedback ausgegeben.

4. Tupel: Die Relationen unterscheiden sich in ihrer Tupel-Menge. Analog zum Schema-Feedback wird hier als Feedback ein falsches Tupel zurückgegeben.

5. Reihenfolge: Die Relationen sind bzgl. ihrer Tupel-Menge identisch, unterscheiden sich aber in ihrer Reihenfolge. Dem Studierenden wird dieser Hinweis als Feedback zurückgegeben. Dieser Schritt wird nur ausgeführt, wenn die Relevanz der Tupel-Reihenfolge in der Aufgabe spezifiziert wurde.

Insbesondere aus den Fehlermeldungen der Schritte 2 bis 4 lassen sich für den Studierenden Hinweise ziehen, um das Ergebnis zu korrigieren. Einfache Fehler, wie z. B. ein vergessenes oder falsch geschriebenes Attribut im SELECT, lassen sich so schnell korrigieren. Durch den konkreten Hinweis auf falsche Attribute bzw. falsche Tupel erhalten die Studierenden insbesondere ein personalisiertes Feedback bzgl. ihrer Lösung und sind tendenziell eher motiviert, im Selbststudium ihre Lösung zu überarbeiten. Natürlich besteht dabei auch die Möglichkeit, die richtige Lösung durch schrittweises Ausprobieren „abzufragen“ und sich der korrekten Lösung ,anzunähern“. Diese Möglichkeit wird aber bewusst in Kauf genommen und wird erfahrungsgemäß von den Studierenden kaum ausgenutzt.

Bewertung und Feedbackgenerierung für das Aufgabenformat SCHEMA verläuft ähnlich zu den oben skizzierten Verfahren. Die Musterlösung wird durch die Angabe eines Tabellennamens definiert, mit dessen Hilfe dann das Schema (Attributname, Primär- und Fremdschlüssel) aus der Datenbank ausgelesen werden. Eine Prüfung auf weitere Constraints wie z. B. Datentypen, NOT NULL oder UNIQUE erfolgt nicht, wäre aber prinzipiell denkbar. Die Feedbackge- 
nerierung erfolgt auch hier wieder abgestuft: zunächst wird die Anzahl der Attribute geprüft, danach die Attributnamen und dann pro Attribut die Angaben zum Primär- bzw. Fremdschlüssel. An jedem Schritt kann das entsprechende Feedback generiert werden, wie z. B. „Falsches Attribut X“ oder „Falsche Primärschlüsselangabe bei Attribut Y“”.

Bei Aufgaben des Ergebnisformats CHECK werden innerhalb der Aufgabe entsprechende Testfälle angegeben. Diese bestehen aus SQL-Statements, die entweder ein definiertes Query-Ergebnis zurückliefern müssen (Feedback analog zu SELECT) oder einen entsprechenden Statusbzw. Fehlercode liefern müssen. Bei letzterem ist das Feedback dann der erwartete Code.

\section{Implementation und Einsatzszenarien}

Die aktuelle DMT-Implementation ist eine Webservice-basierte Webanwendung unter Verwendung von Java-Servlets, HTML und Javascript. Die Funktionalität wird durch zwei REST-basierte Webservices realisiert:

- /gettaskinfo?taskid=... liefert alle zum Anzeigen der Aufgabe notwendigen Informationen (u.a. Aufgabenstellung und Ergebnisformat) sowie den Status (u. a. verfügbare Tabellen) als JSON-Objekt

- /gettaskresult?taskid=. . \&answer=... . liefert Bewertung und Feedback bzgl. der übergebenen Antwort ebenfalls als JSON-Objekt

Die Architektur auf Basis der REST-Schnittstellen ermöglicht eine einfache Integration in verschiedene User Interfaces. In der aktuellen Implementation ist das eine HTML-Seite, die per Javascript mit den Webservices kommuniziert und mittels JQuery dynamisch die Darstellung manipuliert.

Der Quellcode von DMT wird als Open-Source-Projekt auf Github bereitgestellt ${ }^{6}$, das auch eine kurze, technische Beschreibung enthält. Zur einfachen Verwendung enthält das Projekt ein Docker-Compose-File. Mittels dockercompose lassen sich damit sehr einfach der DMT-WebServer inkl. eines zugehörigen Datenbank-Servers (PostgreSQL) starten. Beim Start wird das Datenbanksystem automatisch mit Beispieldaten befüllt, u. a. der Mondial-Datenbank. Die Java-Servlets greifen per JDBC auf die Datenbank zu, um u.a. die Anfrageergebnisse der Musterlösungen sowie der Studierendenlösung einzulesen und innerhalb der Programmlogik zu vergleichen. Für Aufgaben der Typen SCHEMA und CHECK wird zusätzlich auf die Metadaten der Resultset- bzw. Statement-Objekte zugegriffen sowie PostgreSQL-Fehlercodes via Exception Handling ausgewertet.

${ }^{6}$ https://github.com/andreas-thor/dasp-dmt.
DMT wurde in den letzten Semestern von den Autoren an der Hochschule für Technik, Wirtschaft und Kultur (HTWK) Leipzig und der Hochschule Mittweida (HSMW) jeweils in der Datenbank-Lehre in den Bachelor-Studiengängen eingesetzt. Zielgruppe des Einsatzszenarios an der HTWK Leipzig waren Studierende der Telekommunikationsinformatik, die DMT als Werkzeug zur Überprüfung von Übungsaufgaben einsetzen. Dabei wurden Lösungen wie z.B. SQL-Queries in eigenen Werkzeugen oder auf Papier entwickelt und dann für die Bewertung und Feedbackgenerierung in das DMT-System übertragen. Sowohl die manuelle Analyse der anonymisierten Logfiles als auch die Gespräche mit den Studierenden zeigten, dass sie das unmittelbar verfügbare Feedback wertschätzten und zur Überarbeitung ihrer Lösungen verwendeten. Teilweise zeigten die Studierenden sportlichen Ehrgeiz, um unbedingt ein „Lösung ist korrekt" von DMT zu erhalten. In wenigen Ausnahmefällen zeigte sich auch studentischer Ehrgeiz, das System mit den bereits oben beschriebenen Ansätzen auszutricksen. Dieses Beobachtung deckt sich mit den Erfahrungen anderer E-Assessment-Systeme zu automatischen Auswertung von Programm-Code [10].

Demgegenüber wurde DMT an der HS Mittweida vorrangig von Studierenden in Nicht-Informatik-Studiengängen eingesetzt, die SQL als erste „Informatik-Sprache“ erlernen. Die Verwendung mächtiger SQL-Clients bzw. anderweitiger Werkzeuge ist oftmals zu komplex für diese Zielgruppe. Daher entwickelten diese Studierende unter Anleitung des Dozenten SQL-Anfragen direkt mit DMT und profitierten bei syntaktisch korrekten Anfragen von dem Feedback, was zum besseren Verständnis sowie zum schrittweisen Aufbau der Anfrage genutzt wurde.

\section{Verwandte Arbeiten}

In den letzten Jahren wurden einige Systeme zur automatischen Bewertung studentischer Lösungen insbesondere in der Mathematik- und Programmierausbildung entwickelt, die u.a. in dem seit 2013 durchgeführten Workshop „Automatische Bewertung von Programmieraufgaben“ " [11] publiziert werden. Dazu zählen beispielsweise die Kontrolle mathematischer Beweise und die Verifikation von Quellcode in verschiedenen Programmiersprachen (z. B. Java). Freie Systeme, die solche Funktionalitäten anbieten umfassen u. a. EASy [8], MathX ${ }^{3}$ [6] oder den an der Universität Passau entwickelten Praktomaten.

Learning-Management-Systeme wie z.B. Ilias, Moodle oder OLAT bieten häufig über eine Plugin-Schnittstelle die Möglichkeit zu Erweiterungen. Exemplarisch sei für Moodle auf [2] für eine große Auswahl an Plugins im Bereich E-Assessment verwiesen. Weitere Linked-in Lösungen für diese Plattformen wie aSQLg [7, 13] bieten 
die Möglichkeit, Aufgaben mit thematischen Bezug zu Datenbanksystemen und insbesondere zur Anfragesprache SQL zu erstellen und die selbstständig von Studierenden eingestellten Lösungen automatisch nach einer vorgebenen Konfiguration bewerten zu lassen.

Oftmals liegt der Schwerpunkt dieser E-AssessmentSysteme in der Bewertung der von Studierenden spezifizierten Ergebnisse einer Aufgabe, um z.B. in Zusammenhang mit einem Aufgabenblatt oder einer Prüfung eine übergreifende Bewertung der Leistung und ggf. eine Note automatisiert abzuleiten. Darüber hinaus erlauben einige Systeme die Erstellung parametrisierter Aufgaben, so dass Studierende personalisierte Aufgaben bekommen. Im Gegensatz zu DMT kommt die Rückmeldung von personalisiertem Feedback an die Studierenden meist zu kurz.

Im Bereich der Datenbank-Lehre gibt es noch eine Vielzahl weiterer Werkzeuge, die das Lernen der Studierenden unterstützen. Einen Learning-by-Doing Ansatz verfolgt SQL Islands [12]. Ähnlich wie DMT geht es in dem Tool um das individuelle Feedback an die Studierenden. Dazu werden die Aufgaben jedoch in einer Geschichte ,gamifiziert“. Analog zu den SELECT-Aufgaben bei DMT agiert das Tool SQLcoach [4]. Studierende sind aufgefordert, SQL-Anfragen für ein gegebenes Schema zu erstellen. Bei falschen Antworten zeigt SQLcoach neben dem Ergebnis der (falschen) SQL-Anfrage eine Preview (z.B. einen Datensatz) des korrekten Ergebnisses. Der in [9] vorgestellte SQLTester verfolgt einen analogen Ansatz. Auf Basis des protokollierten Nutzungsverhaltens sowie einer Umfrage unter den Studierenden präsentieren die Autoren in [9] eine umfangreiche Analyse, wie häufig Studierende Aufgaben (wiederholt) bearbeiten, wie sie die Nützlichkeit des Werkzeugs für das eigene Lernen bewerten und wie die Übungsergebnisse mit den späteren Prüfungsergebnissen korrelieren.

Das eLearning Portal der TH Köln [1] ist ein Toolset und bietet neben Multiple-Choice Fragen dem Studierenden die Möglichkeit mit speziellen Trainern zu arbeiten, die jeweils einen konkreten Bereich abdecken. Ein SQL Trainer dient dem Erlernen der Anfragesprache SQL, der ER-Trainer bedient die Modellierung mit ER-Diagrammen und der 3NF-Trainer den Bereich der Normalisierung (insbesondere 3NF). Ein SQL Puzzle vermittelt spielerisch Kenntnisse in der Anfragesprache SQL. Speziell für die relationale Algebra wurde an der Universität Innsbruck mit RelaX [3] eine Umgebung zum Entwickeln und Ausführen von Ausdrücken der relationalen Alegbra entwickelt.

\section{Zusammenfassung und Ausblick}

Dieser Artikel stellt das E-Assessment-Tool DMT (Data Management Tester) vor, das in der Datenbank-Lehre so- wohl in Praktika als auch während des Selbststudiums eingesetzt werden kann. DMT unterstützt dazu fünf verschiedene Ergebnisformate mit deren Hilfe eine große Bandbreite typischer Aufgaben im Datenbankbereich abgedeckt werden kann. Studierende erhalten für ihre Lösungen eine automatische Bewertung sowie ein Feedback, welches konkrete Hinweise bzgl. ihrer fehlerhaften Lösung gibt. Damit verfolgt DMT das Ziel, Studierende in ihrem individuellen Lernprozess durch zielgerichtetes Feedback zu animieren, ihre Lösungen kontinuierlich zu verbessern.

Zukünftig sollen weitere Datenmodelle (JSON, XML, Graph) sowie korrespondierende Anfragesprachen wie (u. a. XPath und XQuery) in entsprechenden Ergebnisformaten adressiert werden. Ebenso ist die Unterstützung der LTISchnittstelle (Learning Tools Interoperability) geplant, um DMT in bestehende Lernplattformen integrieren zu können. Zusätzlich wollen die Autoren in den kommenden Semestern Evaluierungen gemeinsam mit den Studierenden vornehmen.

Funding Open Access funding enabled and organized by Projekt DEAL.

Open Access Dieser Artikel wird unter der Creative Commons Namensnennung 4.0 International Lizenz veröffentlicht, welche die Nutzung, Vervielfältigung, Bearbeitung, Verbreitung und Wiedergabe in jeglichem Medium und Format erlaubt, sofern Sie den/die ursprünglichen Autor(en) und die Quelle ordnungsgemäß nennen, einen Link zur Creative Commons Lizenz beifügen und angeben, ob Änderungen vorgenommen wurden.

Die in diesem Artikel enthaltenen Bilder und sonstiges Drittmaterial unterliegen ebenfalls der genannten Creative Commons Lizenz, sofern sich aus der Abbildungslegende nichts anderes ergibt. Sofern das betreffende Material nicht unter der genannten Creative Commons Lizenz steht und die betreffende Handlung nicht nach gesetzlichen Vorschriften erlaubt ist, ist für die oben aufgeführten Weiterverwendungen des Materials die Einwilligung des jeweiligen Rechteinhabers einzuholen.

Weitere Details zur Lizenz entnehmen Sie bitte der Lizenzinformation auf http://creativecommons.org/licenses/by/4.0/deed.de.

\section{Literatur}

1. TH-Köln eLearning Datenbank. https://edb2.gm.th-koeln.de. Zugegriffen: 18. Jan. 2021

2. Liste der Moodle-Plugins im Bereich E-Assessment. https:// moodle.org/plugins/index.php?q=assessment. Zugegriffen: 18. Jan. 2021

3. RelaX - Relational Algebra Calculator. https://dbis-uibk.github.io/ relax/landing. Zugegriffen: 18. Jan. 2021

4. Hochschule Kaiserslautern (Hrsg) SQLcoach. https://sqlcoach. informatik.hs-kl.de. Zugegriffen: 18. Jan. 2021

5. Anderson LW, Krathwohl DR (Hrsg) (2001) A taxonomy for learning, teaching, and assessing. A revision of bloom's taxonomy of educational objectives. Allyn \& Bacon, New York

6. Derr K, Fried T, Hornung B, Saller D (2009) MathX3 - OnlineSelbsttest zur Basiskompetenz Mathematik. Z Hochschulentwickl $4(1): 46-59$

7. Garmann R, Fricke P, Reiser P, Bersuch C, Bott OJ (2017) Moodle, Grappa, aSQLg, Graja - Neue Entwicklungen bei der Grading- 
Software der Hochschule Hannover(New Developments in the Grading Software of the Hanover University of Applied Sciences and Arts). In: Strickroth S, Müller O, Striewe M (Hrsg) Proceedings of the Third Workshop Automatische Bewertung von Programmieraufgaben (ABP)

8. Gruttmann SJ, Böhm D, Kuchen H (2008) E-assessment of Mathematical Proofs: Chances and Challenges for Students and Tutors. In: International Conference on Computer Science and Software Engineering (CSSE)

9. Kleerekoper A, Schofield A (2018) SQL tester: an Online SQL assessment tool and its impact. In: Proceedings of the 23rd annual ACM conference on innovation and technology in computer science education

10. Kratzke N (2019) Smart like a fox: how clever students trick dumb automated programming assignment assessment systems. In: Lane
H, Zvacek S, Uhomoibhi J (Hrsg) Proceedings of the 11th International Conference on Computer Supported Education (CSEDU)

11. (2013) Proceedings of the First Workshop Automatische Bewertung von Programmieraufgaben (ABP). In: Priss U, Striewe M (Hrsg) CEUR Workshop Proceedings, Bd. 1067

12. Schildgen J, Deßloch S (2015) SQL-Grundlagen spielend lernen mit dem Text-Adventure SQL Island. In: 16. GI-Fachtagung Datenbanksysteme für Business, Technologie und Web (BTW), DemoProgramm

13. Stöcker A, Becker S, Garmann R, Heine F, Kleiner C, Bott OJ (2013) Evaluation automatisierter Programmbewertung bei der Vermittlung der Sprachen Java und SQL mit den Gradern aSQLg und Graja aus studentischer Perspektive. In: Breiter A, Rensing C (Hrsg) DeLFI 2013 - Die 11. E-Learning Fachtagung Informatik der Gesellschaft für Informatik e.V. (GI), LNI 